\title{
Systematic Review: Targeting HER2 in Bladder Cancer
}

\author{
Vadim S. Koshkin ${ }^{\mathrm{a}}$, Peter O'Donnell ${ }^{\mathrm{b}}$, Evan Y. Yu ${ }^{\mathrm{c}}$ and Petros Grivas ${ }^{\mathrm{d}, *}$ \\ ${ }^{a}$ Department of Medicine, Division of Hematology and Oncology, University of California \\ San Francisco, CA, USA \\ ${ }^{\mathrm{b}}$ Department of Medicine, Section of Hematology/Oncology, University of Chicago, Chicago, IL, USA \\ ${ }^{\mathrm{c}}$ Department of Medicine, Division of Oncology, Clinical Research Director, Fred Hutchinson Cancer \\ Research Center, University of Washington, Seattle Cancer Care Alliance, Seattle, WA, USA \\ ${ }^{\mathrm{d}}$ Department of Medicine, Division of Oncology, University of Washington, Seattle Cancer Care Alliance, \\ Seattle, WA, USA
}

Received 23 August 2018

Accepted 22 November 2018

\begin{abstract}
.
Background: HER2 (ErbB2) is a receptor of the Human Epidermal Growth Factor Receptor (HER) family whose role in oncogenesis of numerous malignancies is well described. Drugs targeting HER2 are currently approved in breast and gastroesophageal cancers while pan-HER targeting agents are being evaluated in multiple malignancies. HER2 genomic alterations are commonly described in urothelial cancer and multiple trials have assessed the efficacy of anti-HER2 agents in both muscle-invasive and metastatic urothelial carcinoma.

Objective: To review prospective clinical trials of therapeutic agents with HER2-targeting activity in patients with bladder cancer.

Methods: A systematic search of PubMed, ASCO abstracts and Clinicaltrials.gov was performed to identify studies of HER2-targeting agents in bladder cancer. Reported results from prospective trials were reviewed and summarized.

Results: Eleven prospective clinical trials with reported results were identified that investigated activity of trastuzumab, lapatinib, neratinib, afatinib, or autologous cellular immunotherapy, (DN24-02), in various bladder cancer treatment settings. Another 11 prospective trials that include bladder cancer patients and are investigating agents with anti-HER2 activity are currently ongoing or have completed enrollment but do not have published results. The reported clinical trials had variable HER2-positivity inclusion criteria and most did not meet their pre-specified benchmarks of clinical efficacy. A recent afatinib trial in an unselected patient population had promising findings in patients with HER2 and HER3 alterations.

Conclusion: Trials of HER2-targeting agents generally in unselected bladder cancer patients have not shown definitive clinical efficacy. Better patient selection, such as via utilization of next-generation sequencing assays that detect specific genomic alterations, and novel therapy combinations that include HER2-targeting agents (with immunotherapy or other modalities) may lead to improved outcomes in current, ongoing or future trials.
\end{abstract}

Keywords: Bladder cancer, urothelial carcinoma, HER2, ERBB2, HER family, targeted therapy, trastuzumab, afatinib, lapatinib, neratinib

\section{INTRODUCTION}

${ }^{*}$ Correspondence to: Petros Grivas, MD, PhD, Associate Professor, Department of Medicine, Division of Oncology Clinical Director, Genitourinary Cancers Program, University of Washington/SCCA, 825 Eastlake Ave E, MS: G4830, Seattle, WA 98109, USA. Tel.: +1 20660675 95; E-mail: pgrivas@uw.edu.
Bladder cancer is a very common malignancy with 81,190 newly diagnosed cases and over 17,240 deaths from this disease predicted to occur in the United States in 2018 [1]. Among all patients with 
muscle-invasive bladder cancer, the 5-year overall survival is around 50\% and many patients progress to metastatic disease [2]. Locally-advanced, unresectable or metastatic bladder cancers are typically fatal, and the 5-year overall survival rate is usually limited to $10-15 \%$ [3]. The standard of care in patients with advanced bladder cancer has been platinum-based combination chemotherapy, preferentially cisplatin-based regimens for patients who are able to tolerate cisplatin and carboplatin-based regimens for those patients who are ineligible for cisplatin [4-9]. Despite initially high response rates, most patients inevitably progress and succumb to this disease [3]. Recently, immune checkpoint inhibitors have emerged as an additional option in the advanced setting for both front-line cisplatin-ineligible disease (pembrolizumab or atezolizumab) $[10,11]$ and in the salvage, platinum-refractory setting where five agents have achieved regulatory approval [12-16]. Despite the excitement generated by these agents, the objective response rates for advanced disease patients remain modest at $15-21 \%$ for platinumrefractory disease and $23-29 \%$ in the front-line cisplatin-ineligible setting.

Given this context, there is significant interest in incorporating targeted therapies into the treatment of advanced urothelial cancer, including agents targeting the Human Epidermal Growth Factor Receptor (HER; ErbB) family $[17,18]$. This is a receptor family consisting of EGFR (ErbB1), HER2 (ErbB2), HER3 (ErbB3) and HER4 (ErbB4), whose activation results in the downstream activation of signaling pathways that promote cell growth, proliferation and possibly chemotherapy resistance $[19,20]$. HER 2 is unique among these receptors in being able to generate downstream signal without a ligand, whereas other receptors require ligand binding for receptor homo- or heterodimerization that produces downstream effects. Comprehensive molecular profiling as part of The Cancer Genome Atlas (TCGA) and other datasets have demonstrated significant incidence of ErbB family amplifications, mutations and over-expression in bladder cancer [21-24]. These molecular alterations of the ErbB family are thought to occur in up to 20-30\% of bladder cancer patients.

The role of HER2 alterations in particular has captured attention leading to significant interest in agents specifically targeting this receptor in bladder cancer, with particularly high rates of HER 2 alterations noted in micropapillary bladder cancer $[25,26]$. The data on the relationship of HER2 expression with clinical outcomes, however, is mixed [19, 27-29]. At least one study has compared HER2 expression in the primary tumor (28\%) vs. locoregional lymph node metastases (53\%) suggesting that HER2 expression may play a role in the progression of bladder cancer [27]. One of the possible pitfalls of clinical translation of HER2 as both prognostic and predictive biomarker has been the discordance between HER2 immunohistochemistry (IHC) assignment, Fluorescence in situ hybridization (FISH), and genomic-level molecular characterization. FISH positivity rates of HER2 over-expression have generally been lower than IHC positivity rates in the published studies and many studies did not include genomic characterization of HER2 at all. The variability of reported HER2 alterations in many studies additionally likely reflects differences in the tested populations, disease stage, immunohistochemistry assays and cut-off used, as well as inter-tumor and intra-tumor heterogeneity. Despite these pitfalls, the generally high degree of HER2 alterations reported in bladder cancer as well as the availability of HER2-targeting agents encouraged the design of relevant clinical trials. Some of the HER2-targeted agents investigated in clinical trials of bladder cancer have included trastuzumab, lapatinib, afatinib, neratinib, T-DM1 as well as other investigational agents. In this review, we systematically review the available prospective data on HER2-targeting agents in both muscle-invasive and metastatic bladder cancer and discuss future directions of this treatment approach.

\section{METHODS}

We performed a systematic search of PubMed, Clinicaltrials.gov and American Society of Clinical Oncology (ASCO) conference abstracts to identify manuscripts, clinical trials and abstracts relevant to the topic of HER2-targeted therapies in bladder cancer. A PubMed search using permutations of the terms "HER2" with "bladder cancer" and with "urothelial carcinoma" as well as the terms "ERBB2" with "bladder cancer" and with "urothelial carcinoma" was undertaken. The abstracts of these articles were then screened for their relevance to the topic under consideration. Additional articles that were referenced in the relevant publications were also considered. In particular, we focused on prospective clinical trials of HER2-targeting agents in bladder cancer and included all prospective trials with reported efficacy data. To assess the relevant clinical trials, Clinicaltrials.gov was searched with combinations 


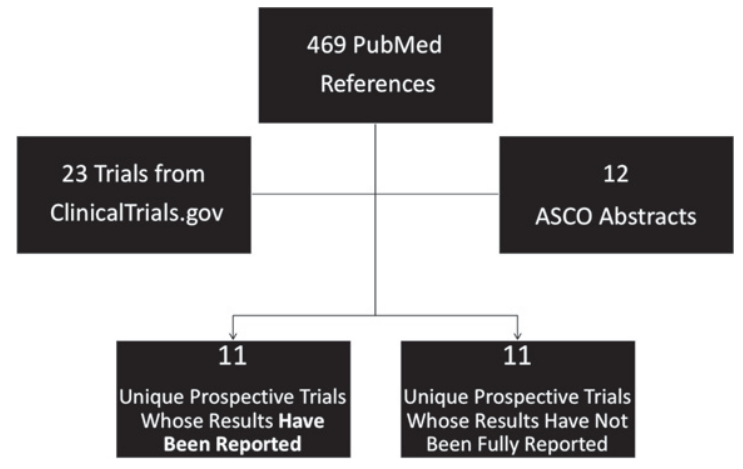

Fig. 1. Schematic for Study Inclusion in Systematic Review of HER2-Targeted Therapies in Bladder Cancer.

of the terms "ERBB2" and "bladder cancer" and "ERBB2" and "urothelial carcinoma." Substituting "HER2" for "ERBB2" in the search generated the same results. Finally, the ASCO database of conference abstracts (https://meetinglibrary.asco.org) was also queried with the combination of terms "ERBB2" and "urothelial carcinoma" and "HER2" and "urothelial carcinoma." Prospective studies were included if they assessed the efficacy of HER2-targeting agents in patients with bladder cancer. Basket trials that included patients with urothelial or bladder cancer were also considered for this review. Trials of HER2-targeted agents with reported results as well as currently ongoing trials were summarized (Fig. 1) Due to the heterogeneous nature of these studies and the variability of HER2-targeting agents used, metaanalysis of the trial results was not performed.

\section{RESULTS}

\section{Overview of search results}

Literature search in PubMed identified 469 published references related to HER2-targeted therapies in bladder cancer which were then screened for relevance based on prospective trial design or the availability of prospective data. The search in ClinicalTrials.gov produced 23 unique trials relevant to this topic. The search in the ASCO database resulted, after screening for reports of prospective trials, in 12 abstracts describing prospective trials of HER2-targeted agents in bladder cancer. A number of these abstracts described results from the same trials as generated from the PubMed search. The remaining abstracts were excluded as they were not specifically focused on treatment or prospective trials.
In the end, a total of 11 prospective trials with reported results were identified and reviewed (Table 1). Another 11 prospective trials are either open to accrual or have finished enrollment but have not yet reported results. The HER2-targeted agents investigated in published prospective trials include trastuzumab, lapatinib, afatinib, neratinib, autologous cellular immunotherapy, DN24-02, and a few more agents. As, the field is dynamic and ever-changing so this review cannot be exhaustive, but a summary of the prospective trials employing HER2-targeting agents is summarized below.

\section{Prospective trials of HER2-targeting agents in muscle-invasive bladder cancer}

Trastuzumab is a monoclonal antibody targeting the HER 2 receptor that preferentially mediates antibody-dependent cellular cytotoxicity of HER2overexpressing tumor cells [30]. It is approved by the United States Food and Drug Administration (FDA) for the treatment of HER2-overexpressing breast cancer in the adjuvant and metastatic settings and HER2-positive gastric and gastroesophageal junction adenocarcinoma in the metastatic setting. In urothelial cancer, most prospective trials of trastuzumab and other HER2-targeting agents have been pursued in the advanced/metastatic setting and mostly for treatment-refractory patients as described in the next section. However, the efficacy of HER2-targeted therapy in muscle-invasive bladder cancer has also been assessed. The NRG Oncology RTOG 0524 trial was a phase $1 / 2$ study of muscleinvasive bladder cancer patients who did not have metastatic disease but were cystectomy-ineligible and treated with bladder-preservation strategy [31]. Patients received radiation (1.8-Gy daily fractions to a total dose of $64.8 \mathrm{~Gy}$ ) in combination with either weekly paclitaxel or weekly paclitaxel and trastuzumab, with therapy assigned based on HER2 overexpression. A total of 20 evaluable patients with $2+$ or $3+$ IHC expression of HER2 were treated with weekly paclitaxel and trastuzumab concurrent with radiation (group 1), while 46 patients with HER2 negative or $1+$ IHC expression were treated with weekly paclitaxel concurrent with radiation (group 2); intended treatment duration was 7 weeks. The primary endpoint was treatment-related toxicity assessed in each group while secondary endpoints included complete response rate, protocol completion rate and overall survival. Group 1 patients had a median age of $79.5,90 \%$ were men, and 
Table 1

Reported Prospective Trials of HER2-targeting Agents in Bladder Cancer

\begin{tabular}{|c|c|c|c|c|c|c|}
\hline $\begin{array}{c}\text { Anti-HER2 Agent } \\
\text { (Year Published } \\
\text { Number of Pts) }\end{array}$ & Treatment setting, Phase & Investigated Treatment & Comparator Arm & $\begin{array}{l}\text { HER2 Expression } \\
\text { Selection Criteria }\end{array}$ & $\begin{array}{l}\text { Response Rate (in exp. } \\
\text { arm) }\end{array}$ & $\begin{array}{l}\text { Median Survival (exp. } \\
\text { arm) }\end{array}$ \\
\hline $\begin{array}{l}\text { Trastuzumab (2016) } \\
\quad \mathrm{N}=66\end{array}$ & $\begin{array}{l}\text { Muscle-invasive, } \\
\text { cystectomy ineligible, } \\
\text { Phase } 1 / 2\end{array}$ & $\begin{array}{l}\text { Trastuzumab, paclitaxel, } \\
\text { RT }\end{array}$ & Paclitaxel/RT & IHC $2+$ and $3+$ & CR (1 yr): $70 \%$ & N/A \\
\hline $\begin{array}{l}\text { DN24-02 (2016) } \\
\quad \mathrm{N}=142\end{array}$ & $\begin{array}{l}\text { Muscle-invasive, } \\
\text { post-surgery, Phase } 2\end{array}$ & DN24-02 & Surveillance & $\mathrm{IHC} \geq 1+$ & N/A & $\begin{array}{l}\text { DRFS: } 11.9 \text { mos OS: } \\
37.0 \text { mos }\end{array}$ \\
\hline $\begin{array}{l}\text { Trastuzumab (2007) } \\
\quad \mathrm{N}=44\end{array}$ & $\begin{array}{l}\text { Metastatic, chemo-Naive } \\
\text { (for met disease) } \\
\text { Phase } 2\end{array}$ & $\begin{array}{l}\text { Trastuzumab, Paclitaxel, } \\
\text { Carboplatin, } \\
\text { Gemcitabine }\end{array}$ & N/A & $\begin{array}{l}\text { IHC } 2+\text { and } 3+, \text { FISH } \\
\text { positive, HER2 ECD }\end{array}$ & ORR: $70 \%$ (11\% CR) & $\begin{array}{l}\text { PFS: } 9.3 \text { mos OS: } \\
\quad 14.1 \mathrm{mos}\end{array}$ \\
\hline $\begin{array}{l}\text { Trastuzumab (2015) } \\
\qquad \mathrm{N}=61\end{array}$ & $\begin{array}{l}\text { Advanced/Metastatic } \\
\text { Chemo-Naive Phase } 2\end{array}$ & $\begin{array}{l}\text { Trastuzumab, } \\
\text { Gemcitabine and } \\
\text { Platinum }\end{array}$ & $\begin{array}{l}\text { Gemcitabine and } \\
\text { Platinum }\end{array}$ & IHC $2+/ 3+$ AND FISH+ & ORR: $53.2 \%(22 \% \mathrm{CR})$ & $\begin{array}{l}\text { PFS: } 8.2 \text { mos OS: } \\
14.1 \mathrm{mos}\end{array}$ \\
\hline $\begin{array}{l}\text { Lapatinib (2008) } \\
\qquad \mathrm{N}=59\end{array}$ & $\begin{array}{l}\text { Metastatic, } \\
\text { platinum-refractory } \\
\text { Phase } 2\end{array}$ & Lapatinib & N/A & $\begin{array}{l}1+, 2+, 3+\text { IHC of either } \\
\text { HER } 2 \text { or EGFR }\end{array}$ & PR: $1.7 \%$ SD: $31 \%$ & $\begin{array}{l}\text { OS: } 4.1 \text { months } \\
\text { (17.9 weeks) }\end{array}$ \\
\hline $\begin{array}{l}\text { Lapatinib (2010) } \\
\qquad \mathrm{N}=9 \text { (bladder pts) }\end{array}$ & $\begin{array}{l}\text { Metastatic, } \\
\text { treatment-refractory } \\
\text { Phase } 2 \text { (rand. discont.) }\end{array}$ & Lapatinib & Placebo & FISH positive & $\begin{array}{l}\text { Initial treatment in } \\
\text { bladder pts ORR: } 0 \% \\
\text { SD: } 33 \%\end{array}$ & N/A \\
\hline $\begin{array}{l}\text { Lapatinib (2016) } \\
\qquad \mathrm{N}=17\end{array}$ & $\begin{array}{l}\text { Metastatic, chemo-naive } \\
\text { Phase } 1 \text { (dose } \\
\text { escalation) }\end{array}$ & $\begin{array}{l}\text { Lapatinib, Gemcitabine, } \\
\text { Cisplatin }\end{array}$ & N/A & None (all comers) & $\begin{array}{l}\text { ORR: } 59 \% \text { (6\% CR) SD: } \\
\quad 23.5 \%\end{array}$ & N/A \\
\hline $\begin{array}{l}\text { Lapatinib (2016) } \\
\qquad \mathrm{N}=15\end{array}$ & $\begin{array}{l}\text { Metastatic, } \\
\text { platinum-refractory, } \\
\text { Phase } 2\end{array}$ & Lapatinib Docetaxel & N/A & None (all comers) & $\begin{array}{l}\text { ORR: } 8 \% \text { ( } 8 \% \text { CR) SD: } \\
\quad 31 \%\end{array}$ & PFS: $2.0 \mathrm{mos}$ OS: $6.3 \mathrm{mos}$ \\
\hline $\begin{array}{l}\text { Lapatinib (2017) } \\
\quad \mathrm{N}=232\end{array}$ & $\begin{array}{l}\text { Metastatic, no PD } \\
\text { following initial chemo } \\
\text { Phase } 3 \text { (maintenance) }\end{array}$ & Lapatinib & Placebo & $\begin{array}{l}\text { 2+, 3+ IHC for HER2 } \\
\text { and/or EGFR }\end{array}$ & N/A & $\begin{array}{l}\text { PFS: } 4.5 \text { mos OS: } \\
12.6 \mathrm{mos}\end{array}$ \\
\hline $\begin{array}{l}\text { Afatinib (2016) } \\
\qquad \mathrm{N}=23\end{array}$ & $\begin{array}{l}\text { Metastatic, } \\
\text { platinum-refractory } \\
\text { (1 line) Phase } 2\end{array}$ & Afatinib & N/A & $\begin{array}{l}\text { None (all comers, but } \\
\text { PCR done in all } \\
\text { patients) }\end{array}$ & $\begin{array}{l}\text { ORR: } 8.6 \% \text { SD: } 30.4 \% \\
\text { Among } 6 \text { pts with } \\
\text { HER2/HER3 } \\
\text { alterations: ORR } 83 \%\end{array}$ & $\begin{array}{l}\text { PFS: } 1.4 \text { mos OS: } 5.3 \mathrm{mos} \\
\text { Among 6pts with } \\
\text { HER2/HER3 } \\
\text { alterations: mPFS } 6.6\end{array}$ \\
\hline
\end{tabular}

*SUMMIT phase 2 trial of neratinib is not included in the above table since details regarding outcomes in bladder cancer patients were not available, aside from reported lack of activity. 
95\% had ECOG performance status $0-1$. The primary endpoint of experiencing a protocol-specified treatment-related adverse event rate was $35.0 \%$ in group 1 and $30.4 \%$ in group 2; most common adverse events were gastrointestinal in nature. Complete response $(\mathrm{CR})$ rates (defined as no gross tumor at cystoscopy, negative biopsy or both by week 12 following completion of protocol treatment) were $61.5 \%$ in group 1 and $62.5 \%$ in group 2, whereas CR rates at one year in evaluable patients were $72.2 \%$ and $67.6 \%$, respectively. Overall, $60 \%$ of patients treated with paclitaxel/trastuzumab and $74 \%$ patients treated with paclitaxel monotherapy completed treatment per protocol; survival data have not yet been reported. Complete response rates in a HER2-expressing population in this trial compared favorably with historical controls and had similar rates of adverse events to prior protocols of bladder-sparing therapy. The complete response rate was comparable in the two groups, although group 1 included patients generally considered to have more aggressive disease. One of the challenges of this trial design is that HER2 high expressers were treated with trastuzumab whereas low expressers were not, which makes it difficult to isolate and interpret trastuzumab's effect in this patient population.

Another adjuvant trial investigated DN24-02, an autologous cellular immunotherapy product designed to stimulate an immune response against HER2 [32]. This product is similar to Sipuleucel-T, which is currently approved in metastatic castration-resistant prostate cancer, in that a fusion protein is utilized to produce an antigen presenting cell which is intended to educate cell-mediated and humoral immunity to target a tumor-specific antigen. In the case of DN24-02 the neoantigen is BA7072, which is a recombinant HER2-derived antigen linked to GMCSF. NeuACT was an open label, randomized, phase II clinical trial investigating adjuvant DN24-02 vs. surveillance in HER2 positive $(\geq 1+\mathrm{IHC})$ urothelial cancer patients with high risk disease $(\geq \mathrm{pT} 2$ or $N+$ ) following surgery. It is notable that this trial design allowed for relatively low HER2 expression for patient eligibility. Patients in the DN24-02 group received infusions every 2 weeks for a total of three infusions. This trial under-accrued and among 142 enrolled patients (out of originally intended 180 patients) after 13.2 months of follow-up, the numerically apparent overall survival (OS) advantage for DN24-02 vs. surveillance was not statistically significant (37.0 vs. 22.2 months, HR 0.96; $p=0.87$ ). Additionally, no differences were noted between the two groups in recurrence-free survival (RFS). Exploratory analyses showed enhanced immunologic responses in the DN24-02 group relative to the surveillance group, including increased antigen presenting cell activation, serum cytokines and antigen-specific T-cell responses as well as increased HER2-derived antigen titers more than a year after treatment. Despite the overall negative results of this trial, interpretation of the efficacy of this treatment was limited by the small sample size. Evidence of DN24-02-induced immune response in the treatment arm and numerical difference in OS in this under-powered study suggested potential utility of additional investigation of this agent.

\section{Prospective trials of HER2-targeting agents in advanced and metastatic urothelial cancer}

\section{Trastuzumab}

Trastuzumab was suggested to have clinical efficacy in advanced urothelial cancer based on early case series $[33,34]$. To further investigate the efficacy of trastuzumab in HER2-overexpressing urothelial cancer, a prospective clinical trial was undertaken and published in 2007 [35]. This was a multicenter phase II trial which included patients with HER2 overexpression (IHC $2+$ or $3+$, FISH positive, or elevated serum Her-2/neu extracellular domain (ECD)). Among 109 initially screened patients without prior chemotherapy for metastatic disease, 57 were found to be HER2-positive by at least one method (most by IHC or FISH). Patients with HER2 positive disease had more metastatic sites and more visceral metastases. Trastuzumab was administered at initial loading dose of $4 \mathrm{mg} / \mathrm{kg}$ and at $2 \mathrm{mg} / \mathrm{kg}$ for subsequent doses, and was given on days 1, 8, 15 of 21-day treatment cycles. Patients also received carboplatin (AUC 5) and paclitaxel $\left(200 \mathrm{mg} / \mathrm{m}^{2}\right.$ ) on day 1 , and gemcitabine on days 1 and 8 (800 $\mathrm{mg} / \mathrm{m}^{2}$ ) (non-cisplatin regimen). The primary endpoint was the cardiac toxicity rate while secondary endpoints included toxicity, response rate, time to progression and overall survival. Median number of treatment cycles administered was 6 (range 1-12) in 44 treated patients. There were higher than expected rates of cardiac toxicity in this trial $(22.7 \%$ all grades, $4.5 \%$ Grade 3 ). Additionally, there were 2 therapyrelated deaths. Objective response rate was 70\% (31 of 44 patients) including 5 complete and 26 partial responses, while $57 \%$ of responses were confirmed. The median time to progression was 9.3 months and median overall survival 14.1 months. This trial 
showed the trastuzumab with chemotherapy combination to be feasible although the higher than expected rate of cardiac toxicity was concerning. Further assessment of the contribution of trastuzumab to such a chemotherapy regimen would require a randomized clinical trial.

A trial comparing the combination of trastuzumab with gemcitabine/platinum vs. gemcitabine/platinum alone in in advanced urothelial carcinoma overexpressing HER2 was published in 2015 [36]. This was a phase II trial that included only patients who were HER2-positive by IHC $(2+$ or $3+)$ and confirmed by FISH. Among 563 screened patients, 75 were HER2-positive by these criteria, and among them, 61 also met the remaining eligibility criteria and were randomized to one of two treatment arms. Arm A received gemcitabine $\left(1000 \mathrm{mg} / \mathrm{m}^{2}\right)$ on day 1 and day 8 and either cisplatin $\left(70 \mathrm{mg} / \mathrm{m}^{2}\right)$ or carboplatin $(\mathrm{AUC}=5)$ on day 1 of a 21 -day cycle. Arm B received the same regimen plus trastuzumab given at a higher dose than in the previous trial ( 8 $\mathrm{mg} / \mathrm{kg}$ loading dose followed by $6 \mathrm{mg} / \mathrm{kg}$ every 21 days). Given the low incidence of HER 2 overexpression the number of patients in each treatment arm was low with 29 patients in arm A and 32 patients in arm B. There was no significant difference among the arms in the primary endpoint of median progression-free survival (PFS), which was 10.2 and 8.2 months in arms A and B, respectively. Objective response rate $(65.5 \%$ vs $53.2 \%, p=0.39)$ and median overall survival (15.7 months vs 14.1 months, $p=0.684$ ) similarly were not significantly different among the arms. Patients in the trastuzumab arm who received cisplatin-based chemotherapy had superior outcomes relative to patients with carboplatin-based chemotherapy in an exploratory analysis. In terms of adverse events, there was an incidence of grade 3 cardiotoxicity in the trastuzumab-treated arm, but by far the most common grade 3/4 toxicity in either arm was myelosuppression; another patient in arm B died of febrile neutropenia. Although the trastuzumab combination with chemotherapy was generally well tolerated in this trial, the small sample size may have precluded the detection of significant response and outcome differences.

\section{Lapatinib}

Lapatinib is a tyrosine kinase inhibitor of the intracellular domains of EGFR and HER2 receptors that is currently FDA approved as part of combination regimens in metastatic breast cancer [37]. The activity of lapatinib in metastatic urothelial carcinoma was assessed in a single arm phase 2 study that enrolled patients in 29 European centers [38]. This clinical trial included patients whose disease had progressed on prior platinum-based chemotherapy and whose tumors had confirmed $1+, 2+$ or $3+$ IHC expression of either EGFR or HER2. Patients received lapatinib monotherapy at a dose of $1,250 \mathrm{mg}$ daily until disease progression or unacceptable toxicity with response evaluations performed every 8 weeks according to RECIST criteria. The primary endpoint was objective response rate (ORR), but among 59 enrolled patients only 34 were evaluated for response by independent review. Only 1 (3\%) partial response was observed, while $31 \%$ of patients achieved stable disease. Median time to disease progression was 8.6 weeks while median overall survival was 17.9 weeks. Higher rates of clinical benefit (partial response and stable disease) and longer median OS were observed in patients whose tumors overexpressed EGFR and HER2. Most common grade 3/4 events with lapatinib treatment included vomiting (7\%), diarrhea (3\%), dehydration (3\%) and hypernatremia (3\%). Although the trial did not meet its primary endpoint which was ORR $>10 \%$, improved outcomes among 'higher expressers' of EGFR and HER2 were considered promising enough to warrant further investigation of lapatinib in this patient population. It should be noted that since lapatinib targets both HER2 and EGFR, it is not apparent from this trial whether the observed effect was due to the therapy's impact on either one or both of these targets.

A randomized discontinuation study of lapatinib in patients with HER2-amplified solid tumors was also pursued [39]. As part of this novel trial design, patients with HER2 amplified (FISH positive) gastroesophageal, bladder, ovarian or uterine tumors were enrolled into a randomized discontinuation study of lapatinib. Patients were initially administered open label lapatinib $1,500 \mathrm{mg}$ PO daily for 12 weeks. Patients with at least stable disease at the end of this treatment period were then intended to be randomized in a double-blind, 1:1 fashion to either continue lapatinib $1,500 \mathrm{mg} /$ day or receive placebo. The plan was to randomize 100 patients with stable disease at week 12 to lapatinib or placebo. The intended primary endpoint was going to be progression-free survival 12 weeks after randomization. Unfortunately, this trial suffered from low accrual. Among 32 patients with HER2-amplified tumors enrolled, there were 9 patients with metastatic bladder cancer. In the overall cohort, at the end of the initial 12 weeks, only 1 patient had complete response and 9 had stable dis- 
ease. Among the 9 patients with bladder cancer, no responses were noted, however 3 patients had stable disease as best response. From the overall cohort only 7 patients underwent randomization to placebo or lapatinib and the low accrual consequently led to early study closure.

Subsequent lapatinib trials in bladder cancer investigated its efficacy as part of combination regimens or as maintenance therapy following front-line treatment. EORTC 30061 was a phase 1 trial combining lapatinib with gemcitabine and cisplatin in advanced urothelial cancer [40]. The primary aim was to determine the maximum tolerated dose of lapatinib in this combination. This trial used a $3+3$ dose escalation protocol with lapatinib doses of $750 \mathrm{mg}, 1,000 \mathrm{mg}$ and $1,250 \mathrm{mg}$ daily (cohorts $1-3$ ) combined with gemcitabine $\left(1000 \mathrm{mg} / \mathrm{m}^{2}\right.$ on days $\left.1,8,15\right)$ and cisplatin $\left(70 \mathrm{mg} / \mathrm{m}^{2}\right.$ on day 2$)$ given over 28 -day cycles. A total of 17 patients, unenriched for EGFR or HER2 expression, were treated. There were no dose limiting toxicities in cohorts 1 and 2, while in cohort 3 only 1 of 6 patients had grade 4 febrile neutropenia and renal failure. After median follow-up of 6.5 months, the ORR was 59\% (1 complete and 9 partial responses), and 5 of the responses occurred at the highest lapatinib dose. Based on these findings, 1,250 $\mathrm{mg}$ of lapatinib daily was determined the maximally tolerated dose in combination with gemcitabine and cisplatin.

A single-institution phase 2 study assessed the efficacy of the combination of docetaxel and lapatinib in metastatic urothelial carcinoma [41]. This study enrolled metastatic urothelial carcinoma patients previously exposed to platinum-based chemotherapy not selected based on HER2 expression. The study used a Simon's two-stage design that included $14+26$ patients. Initially, 6 patients were administered lapatinib $1,250 \mathrm{mg}$ daily with docetaxel $60 \mathrm{mg} / \mathrm{m}^{2}$ every 3 weeks; subsequent patients received the same dose of lapatinib with docetaxel $75 \mathrm{mg} / \mathrm{m}^{2}$ every 3 weeks. Among the first 15 accrued patients, PFS at 12 weeks was $40 \%$, and although this was on the border of pre-determined efficacy cutoff for the first stage of this trial, trial enrollment was terminated. Best 'RECIST' responses among evaluable patients included 1 patient with complete response (8\%), 4 patients with stable disease (31\%), and 10 patients with progressive disease (62\%). In the entire cohort, median PFS and OS were 2.0 months and 6.3 months, respectively. Common significant adverse events included diarrhea (G3 33\%), vomiting (G3 $27 \%$ ), nausea (G3 27\%) and fatigue (G3 7\%). Based on these results, the lapatinib and docetaxel combination was not recommended for further development in metastatic platinum-refractory urothelial cancer.

The efficacy of lapatinib as maintenance therapy following completion of chemotherapy in patients with EGFR- or HER2-positive metastatic urothelial carcinoma was assessed in a large, randomized, double-blind phase 3 trial [42]. As part of this trial, EGFR and HER2 status was screened centrally in patients with metastatic urothelial carcinoma and $2+$ or $3+$ IHC was considered positive. Patients who screened positive and did not have progression following 4-8 cycles of initial chemotherapy were randomized 1:1 to receive lapatinib $1,500 \mathrm{mg}$ daily or placebo. A total of 232 patients were enrolled and primary endpoint was PFS. Median PFS in the lapatinib and placebo groups was 4.5 and 5.1 months, respectively (HR $1.07 ; 95 \%$ CI 0.81 to $1.43 ; P=.63$ ). Median overall survival with lapatinib and placebo was 12.6 and 12.0 months, respectively (HR 0.96; $95 \%$ CI 0.70 to $1.31 ; P=.80$ ). Predefined subset analyses likewise showed no PFS benefit of lapatinib vs. placebo for patients who were 3 + for EGFR or HER2, positive for EGFR only or positive for HER2 only. Lapatinib was well tolerated and no significant differences were found between lapatinib and placebo arms in terms of grade 3/4 adverse events. Overall, maintenance lapatinib did not show clinical benefit in this setting. Outcome results from the screened population in this trial additionally allowed for a robust prospective analysis showing that EGFR and HER2 status in urothelial cancer was not prognostic, at least based on IHC determination of protein expression.

\section{Afatinib}

Afatinib is an ERBB family inhibitor that covalently binds to the kinase domains of EGFR, HER2, and HER4 and irreversibly inhibits tyrosine kinase autophosphorylation resulting in the downregulation of ErbB signaling [43]. Afatinib is currently approved in EGFR-mutated metastatic non-small cell lung cancer and was hypothesized to have clinical activity in urothelial carcinoma given the frequency of EGFR and HER2 alterations in the latter. The activity of afatinib in metastatic urothelial cancer was investigated in a phase 2 , single arm, open label trial of 23 patients [44]. This trial included patients who had progressed on prior platinum-based chemotherapy and did not require tumors to have ErbB alterations. Following enrollment, patients received afatinib 40 $\mathrm{mg} /$ day until progression or unacceptable toxicity. The primary endpoint was 3-month progression-free 
survival; ErbB alterations (EGFR, HER2, HER3, HER4) as predictors of afatinib sensitivity were also assessed. Overall, 5 out of 23 patients (21.7\%) achieved 3-month PFS and consequently the criterion for enrolling 10 more patients was not met (null hypothesis was 3-month PFS rate of $<30 \%$ ). In the entire cohort, PFS was 1.4 months and ORR was $8.6 \%$. Best responses were PR in 2 patients $(8.6 \%)$ and SD in 7 patients $(30.4 \%)$; median OS for all patients was 5.3 months. Despite this overall low response rate in this unselected patient population, molecular alterations in HER2 and HER3 were associated with increased efficacy of afatinib treatment. Copy number analysis was performed using polymerase chain reaction (PCR) and confirmed with FISH. IHC was also performed separately. Overall, among 21 patients with available tumor samples, 5 had EGFR amplification, 4 had HER2 amplification and 3 had HER3 somatic mutations. All 5 patients who had PFS of at least 3 months had either HER2 amplification or HER3 mutation (one patient had both). Three of four patients with HER2 amplification had PFS of at least 3 months and only one patient with HER2 amplification did not. Interestingly, IHC results for any of the three targets (EGFR, HER2, HER3) had no correlation with outcomes. HER2 IHC results also had low concordance with PCR and FISH. Overall, the median time to progression was 6.6 months in patients with HER2/HER3 alteration and only 1.4 months in patients without alteration $(p<0.001)$. Based on the results of this trial, afatinib is undergoing further investigation in molecularly selected advanced urothelial cancer patients with HER2 or HER3 alteration (NCT02780687).

\section{Neratinib}

Neratinib is another oral pan-HER inhibitor that irreversibly inhibits tyrosine kinase activity of EGFR, HER2 and HER4 leading to reduced activation of downstream signaling pathways [45]. It is currently approved as adjuvant treatment in HER2-overexpressing breast cancer [46] and is currently being tested in clinical trials in a number of other malignancies with described HER2 alterations, including lung, colorectal and bladder. An ongoing phase II 'SUMMIT' basket trial among patients with solid malignancies harboring HER2/HER3 mutation included patients with urothelial carcinoma [47]. Patients were treated with neratinib $240 \mathrm{mg}$ daily and the primary endpoint was ORR per RECIST v1.1 at week 8 following treatment initiation. Only tumor types with 8 -week ORR $\geq 30 \%$ were then investigated further in expansion cohorts. Although responses were reported in breast, biliary tract, salivary, NSCLC and cervical cancers, the activity of neratinib in urothelial cancer was limited and was not pursued further [48].

\section{Currently ongoing trials of HER2-targeting agents in bladder cancer}

In addition to the trials with reported results described above, a number of clinical trials of HER2-targeting agents in urothelial carcinoma are currently ongoing. Large 'basket' genomic biomarker-based trials including NCI-MATCH [49] and MyPathway [50] both include treatment arms of HER2-targeted agents which have enrolled urothelial cancer patients with tumors with HER2 alteration. NCI-MATCH included among its multiple arms, three $\operatorname{arms}(\mathrm{B}, \mathrm{Q}, \mathrm{J})$ treated with agents having anti-HER2 activity (afatinib, T-DM1, trastuzumab with pertuzumab, respectively). Arm J treated with trastuzumab/pertuzumab combination is still open to accrual and preliminary results are awaited. Preliminary results for metastatic urothelial cancer patients on the MyPathway trial were presented in abstract form in 2017 [51]. In this trial, patients with platinumresistant, HER2-positive (HER2 amplification or activating mutations by gene sequencing, FISH or IHC), metastatic urothelial cancer received standard doses of pertuzumab and trastuzumab until progression or unacceptable toxicity. Investigator-assessed ORR according to RECIST (v1.1) was the primary endpoint. Among the first 12 patients treated there was 1 complete response, 2 partial responses and 2 patients with stable disease for at least 4 months. The complete response was durable in a patient with peritoneal metastases. All responses were in patients with tumors with HER 2 amplification and strongly support further evaluation of the trastuzumab/pertuzumab combination in this population.

A number of other trials are currently enrolling urothelial carcinoma patients with HER2 alterations. These trials are investigating both novel agents previously not tested in this setting as well as agents, such as afatinib, in novel settings and combinations. One novel agent is ado-trastuzumab emtansine, (T-DM1), an antibody-dug conjugate targeted against HER2 that is currently FDA approved in HER2-positive metastatic breast cancer. Preclinical data supported the efficacy of T-DM1 in in vitro and in vivo models of HER2-overexpressing bladder cancer cells [52]. As previously noted, it was also investigated in one of 
the arms of the NCI-MATCH trial, although results are not yet available. A small trial of patients with HER2-overexpressing solid tumors, that included a cohort of urothelial carcinoma, treated with TDM1 at doses of $2.4 \mathrm{mg} / \mathrm{kg}$ and $3.6 \mathrm{mg} / \mathrm{kg}$ every 3 weeks recently finished enrollment (NCT02999672) [53]. Another phase 2 trial enrolled patients with HER2-amplified or HER2-mutant cancers, including urothelial cancer, treated with $3.6 \mathrm{mg} / \mathrm{kg}$ of T-DM1 every 21 days (NCT02675829) [54]. Among 3 evaluable patients with urothelial carcinoma there were no responders, however 2 patients had stable disease as best response according to 'RECIST' [55]. Another novel antibody-drug conjugate is ADCT-502, which is trastuzumab conjugated to tesirine, a DNA crosslinking pyrrolobenzodiazepine (PBD) dimer-based drug. A phase I dose-escalation study to evaluate the safety and antitumor activity of this agent in advanced solid tumors with HER2 expression is ongoing (NCT03125200) [56]. A combination of trastuzumab deruxtecan (DS-8201a), another HER2targeting antibody-drug conjugate, with nivolumab is also being investigated in a phase $1 \mathrm{~b}$ clinical trial of patients with HER2-expressing breast and urothelial cancers (NCT03523572) [57]. DS-8201 a has demonstrated significant activity in heavily pre-treated breast and gastric cancer patients and currently has breakthrough designation for metastatic breast cancer patients previously treated with trastuzumab, pertuzumab and T-DM1. This clinical trial, which began enrolling patients in the summer of 2018, includes a dose escalation phase of DS-8201a and dose expansion cohorts based on tumor type and HER2 expression. Patients with advanced urothelial cancer that have progressed on prior platinum-based chemotherapy are being enrolled into one of two cohorts with either IHC $1+$ or IHC $2+/ 3+$ HER2 expression that is centrally determined.

Other novel agents are also being investigated in early phase clinical trials. PRS-343, a CD137/HER2 bispecific compound is being studied in a phase I trial of HER2-positive metastatic solid tumors (NCT03330561) [58]. A therapeutic cancer vaccine ETBX-021, which leads to production of cytotoxic T-cells against HER2 will be investigated in HER2positive urothelial carcinoma patients who had progressed on prior chemotherapy and anti-PD1/PDL1 agents (NCT03197571) [59]. Finally, 2 trials are investigating the efficacy of afatinib in the metastatic, treatment-refractory setting. NCT02780687 is a phase 2 single arm trial of afatinib monotherapy in urothelial carcinoma patients including those with
HER mutation or amplification who have progressed on prior platinum-based therapy [60]. NCT02795156 is a phase 2 study which includes an arm with afatinib (also another with regorafenib against other molecular targets) for patients with EGFR, HER2 or HER4 altered (based on next-generation sequencing), treatment-refractory solid tumors including urothelial carcinoma [61].

\section{DISCUSSION AND FUTURE DIRECTIONS}

HER2 alterations are common in urothelial cancer, and consequently HER 2 has been targeted by numerous agents in multiple prospective clinical trials. At this time, no HER2-targeting agent is approved for urothelial carcinoma. Although significant efficacy of HER2-targeting agents was seen in trials of breast and gastroesophageal cancers, clinical trials in urothelial cancer have generally been negative, which could be explained by a number of reasons. The small sample size and low accrual in many trials potentially made clinically meaningful efficacy signals difficult to detect. The question remains in regard to which is the most efficacious anti-HER2 agent and the optimal targeting modality (monoclonal antibody, tyrosine kinase inhibitor, vaccine or other) at least in urothelial cancer. Single agent, sequential vs. combination therapy is another pragmatic query, as well as the optimal disease state/setting for treatment. Of greatest importance, however, is determining which is the best method of biomarker-based patient selection. Given the variability of HER 2 status across tumor types, the heterogeneity within a particular tumor and clonal evolution, a uniform standard for what qualifies as HER2-positive tumor can help in selecting patients most likely to respond to this targeted therapy. Not all clinical trials described in this review selected patients based on HER2 status, whereas those that did mostly used IHC and occasionally FISH to define HER2 positivity. It is notable that most patients in the trial with afatinib who derived the greatest clinical benefit had HER2/HER3 amplification and/or mutation detected by PCR. HER2 IHC had low concordance with PCR and limited correlation with outcomes in this trial. Although these observations were again limited by the small sample size, they are nevertheless suggestive that next-generation sequencing may be even more relevant in the selection of patients, as noted with other tumors, therapeutic targets and biomarkers. Additionally, many of the investigated agents targeting HER2 
may have additional targets or mechanisms of action, which can make it challenging to determine whether the potential efficacy, or lack thereof, is specifically due to HER2 targeting. This is even more difficult in the context of molecular redundancy in the tumor tissue and within cellular sub-networks.

Considering the significant tumor heterogeneity and dynamic nature of urothelial malignancies, incorporation of both tumor tissue and cell-free circulating tumor DNA (ctDNA) next-generation sequencing might provide additive and complementary information and support further understanding of de novo and acquired resistance mechanisms $[62,63]$. Cellfree ctDNA assessment may increasingly become an important tool in identifying patients that are eligible for clinical trials or standard treatment with HER2 targeting agents. Additionally, ctDNA assessment may eventually be utilized as a dynamic biomarker throughout the course of treatment, with changes in HER2 status informing and tailoring therapy. Further prospective validation is required before ctDNA is ready for routine use in clinical practice; however, its potential use for eligibility and stratification in clinical trials may be worth evaluating at this point. The incorporation of ctDNA as exploratory endpoint within the next generation of clinical trials of HER 2 targeting agents would be an important step in that direction. Moreover, the variety of potential clinical trial designs provides the opportunity for both biomarker discovery and validation; the ultimate question is whether the use of the biomarker can help select patients who derive more benefit from this targeted therapy. However, this "enrichment" approach has to be robustly tested in the appropriate trial setting.

It is also notable that some of the more recently investigated agents with anti-HER 2 activity, such as afatinib, have shown more promise and may eventually show benefit in ongoing and future trials. Going forward, optimized selection of patients whose tumors have HER2 amplification or mutation may be relevant in the peri-operative (neoadjuvant, adjuvant) and advanced disease settings, and/or in bladder preservation approach. HER2-targeted agents can potentially play an important role either as monotherapy or in combination with immune checkpoint inhibitors and/or other agents. Two of the novel drugs currently in development that have received breakthrough designation in bladder cancer are erdafitinib and enfortumab vedotin, which are both molecularlytargeted agents. They exemplify a new trend of utilizing a molecularly targeted approach in treating this heterogeneous disease, and a departure from a "one size fits all" approach that has been generally employed in bladder cancer until the present time. Given the prevalence of HER2 alterations in this disease, the hope is that HER2-targeting agents will also find a niche within molecular stratification algorithms in advanced bladder cancer. With proper biomarker discovery and validation and well-designed clinical trials, anti-HER2 therapy may well join the growing armamentarium of strategies utilized against urothelial cancer in the near future.

\section{CONFLICT OF INTEREST}

Dr. Koshkin has no COI. Dr. Yu reports grants from Agensys, grants from Genentech, grants from Merck, grants from Seattle Genetics; personal fees from Amgen, personal fees from AstraZeneca, grants and personal fees from Bayer, personal fees from Churchill Pharma, grants and personal fees from Dendreon, personal fees from EMD Serono, personal fees from InCyte, personal fees from Janssen, grants and personal fees from Merck, personal fees from Pharmacyclics, personal fees from QED, personal fees from Tolmar Pharmaceuticals, grants and personal fees from Seattle Genetics. Dr. O’Donnell has stocks with Allergan and PrescriptIQ, personal fees from Genentech, Merck, Astra Zeneca, Astellas, Seattle Genetics, Inovio, Janssen, Parexel, Kantar Health, Harrison Consulting Group, Quintiles, OncLive; research funding from Boehringer Ingelheim, Merck, Genentech, AstraZeneca/MedImmune, Acerta Pharma, Janssen, Seattle Genetics, BristolMyers Squibb; he was named as co-inventor on a pending patent for a genomic prescribing system for medication prescribing; has other relationship with Advance Medical and Janssen. Dr. Grivas has done consulting with Genentech, Merck, Janssen, Seattle Genetics, Bristol-Myers Squibb, Astra Zeneca, Clovis Oncology, Biocept, Pfizer, EMD Serono, Foundation Medicine, Driver Inc., QED Therapeutics, Heron; has participated in an unbranded, educational program with Bristol-Myers Squibb; his institutions have had research funding from Genentech, Merck, Mirati, Oncogenex, Bayer, Pfizer, Astra Zeneca, Clovis Oncology, Bavarian Nordic, Immunomedics.

\section{REFERENCES}

[1] Siegel RL, Miller KD, Jemal A. Cancer statistics, 2018. CA. Cancer J Clin. 2018;68:7-30. 
[2] Advanced Bladder Cancer (ABC) Meta-analysis Collaboration. Neoadjuvant chemotherapy in invasive bladder cancer: Update of a systematic review and meta-analysis of individual patient data advanced bladder cancer (ABC) meta-analysis collaboration. Eur Urol. 2005;48:202-5; discussion 205-206.

[3] von der Maase $\mathrm{H}$, et al. Long-term survival results of a randomized trial comparing gemcitabine plus cisplatin, with methotrexate, vinblastine, doxorubicin, plus cisplatin in patients with bladder cancer. J Clin Oncol Off J Am Soc Clin Oncol. 2005;23:4602-8.

[4] Sternberg CN, et al. M-VAC (methotrexate, vinblastine, doxorubicin and cisplatin) for advanced transitional cell carcinoma of the urothelium. J Urol. 1988;139:461-9.

[5] Loehrer PJ, et al. A randomized comparison of cisplatin alone or in combination with methotrexate, vinblastine, and doxorubicin in patients with metastatic urothelial carcinoma: A cooperative group study. J Clin Oncol Off J Am Soc Clin Oncol. 1992;10:1066-73.

[6] Sternberg CN, et al. Randomized phase III trial of highdose-intensity methotrexate, vinblastine, doxorubicin, and cisplatin (MVAC) chemotherapy and recombinant human granulocyte colony-stimulating factor versus classic MVAC in advanced urothelial tract tumors: European Organization for Research and Treatment of Cancer Protocol no. 30924. J Clin Oncol Off J Am Soc Clin Oncol. 2001;19:2638-46.

[7] von der Maase $\mathrm{H}$, et al. Gemcitabine and cisplatin versus methotrexate, vinblastine, doxorubicin, and cisplatin in advanced or metastatic bladder cancer: Results of a large, randomized, multinational, multicenter, phase III study. J Clin Oncol Off J Am Soc Clin Oncol. 2000;18:3068-77.

[8] Galsky MD, et al. A consensus definition of patients with metastatic urothelial carcinoma who are unfit for cisplatinbased chemotherapy. Lancet Oncol. 12:211-4.

[9] De Santis M, et al. Randomized phase II/III trial assessing gemcitabine/carboplatin and methotrexate/carboplatin/ vinblastine in patients with advanced urothelial cancer who are unfit for cisplatin-based chemotherapy: EORTC study 30986. J Clin Oncol Off J Am Soc Clin Oncol. 2012;30: 191-9.

[10] Balar AV, et al. Atezolizumab as first-line treatment in cisplatin-ineligible patients with locally advanced and metastatic urothelial carcinoma: A single-arm, multicentre, phase 2 trial. Lancet Lond Engl. 2017;389:67-76.

[11] Balar AV, et al. First-line pembrolizumab in cisplatinineligible patients with locally advanced and unresectable or metastatic urothelial cancer (KEYNOTE-052): A multicentre, single-arm, phase 2 study. Lancet Oncol. 2017, doi:10.1016/S1470-2045(17)30616-2

[12] Rosenberg JE, et al. Atezolizumab in patients with locally advanced and metastatic urothelial carcinoma who have progressed following treatment with platinum-based chemotherapy: A single-arm, multicentre, phase 2 trial. Lancet Lond Engl. 2016;387:1909-20.

[13] Bellmunt J, et al. Pembrolizumab as Second-Line Therapy for Advanced Urothelial Carcinoma. N Engl J Med. 2017; 376:1015-26.

[14] Massard C, et al. Safety and Efficacy of Durvalumab (MEDI4736), an Anti-Programmed Cell Death Ligand-1 Immune Checkpoint Inhibitor, in Patients With Advanced Urothelial Bladder Cancer. J Clin Oncol Off J Am Soc Clin Oncol. 2016;34:3119-25.

[15] Sharma P, et al. Nivolumab in metastatic urothelial carcinoma after platinum therapy (CheckMate 275): A mul- ticentre, single-arm, phase 2 trial. Lancet Oncol. 2017;18: 312-22.

[16] Apolo AB, et al. Avelumab, an Anti-Programmed DeathLigand 1 Antibody, In Patients With Refractory Metastatic Urothelial Carcinoma: Results From a Multicenter, Phase Ib Study. J Clin Oncol Off J Am Soc Clin Oncol. 2017;35: 2117-24.

[17] Mooso BA, et al. The role of EGFR family inhibitors in muscle invasive bladder cancer: A review of clinical data and molecular evidence. J Urol. 2015;193:19-29.

[18] Rowinsky EK, The erbB family: Targets for therapeutic development against cancer and therapeutic strategies using monoclonal antibodies and tyrosine kinase inhibitors. Annu Rev Med. 2004;55:433-57.

[19] Chow NH, Chan SH, Tzai TS, Ho CL, Liu HS. Expression profiles of ErbB family receptors and prognosis in primary transitional cell carcinoma of the urinary bladder. Clin Cancer Res Off J Am Assoc Cancer Res. 2001;7:1957-62.

[20] Baselga J, Swain SM. Novel anticancer targets: Revisiting ERBB2 and discovering ERBB3. Nat Rev Cancer. 2009;9:463-75.

[21] Cancer Genome Atlas Research Network. Comprehensive molecular characterization of urothelial bladder carcinoma. Nature. 2014;507:315-22.

[22] Iyer $\mathrm{G}$, et al. Prevalence and co-occurrence of actionable genomic alterations in high-grade bladder cancer. J Clin Oncol Off J Am Soc Clin Oncol. 2013;31:3133-40.

[23] Robertson AG, et al. Comprehensive Molecular Characterization of Muscle-Invasive Bladder Cancer. Cell. 2017; 171:540-556.e25.

[24] Ross JS, et al. Comprehensive genomic profiling of 295 cases of clinically advanced urothelial carcinoma of the urinary bladder reveals a high frequency of clinically relevant genomic alterations. Cancer. 2016;122:702-11.

[25] Ross JS, et al. A high frequency of activating extracellular domain ERBB2 (HER2) mutation in micropapillary urothelial carcinoma. Clin Cancer Res Off J Am Assoc Cancer Res. 2014;20:68-75.

[26] Isharwal S, et al. Intratumoral heterogeneity of ERBB2 amplification and HER2 expression in micropapillary urothelial carcinoma. Hum Pathol. 2018;77:63-9.

[27] Jimenez RE, et al. Her-2/neu overexpression in muscleinvasive urothelial carcinoma of the bladder: Prognostic significance and comparative analysis in primary and metastatic tumors. Clin Cancer Res Off J Am Assoc Cancer Res. 2001;7:2440-7.

[28] Gandour-Edwards R, et al. Does HER2/neu expression provide prognostic information in patients with advanced urothelial carcinoma? Cancer. 2002;95:1009-15.

[29] Krüger S, et al. HER2 overexpression in muscle-invasive urothelial carcinoma of the bladder: Prognostic implications. Int J Cancer. 2002;102:514-8.

[30] Trastuzumab. United States Food and Drug Administration, 2018.

[31] Michaelson MD, et al. A Phase 1/2 Trial of a Combination of Paclitaxel and Trastuzumab With Daily Irradiation or Paclitaxel Alone With Daily Irradiation After Transurethral Surgery for Noncystectomy Candidates With MuscleInvasive Bladder Cancer (Trial NRG Oncology RTOG 0524). Int J Radiat Oncol Biol Phys. 2017;97:995-1001.

[32] Bajorin DF, et al. Phase 2 trial results of DN24-02, a HER2-targeted autologous cellular immunotherapy in HER2+urothelial cancer patients (pts). J Clin Oncol. 2016; $34: 4513$. 
[33] Peyromaure M, et al. Trastuzumab (Herceptin) in metastatic transitional cell carcinoma of the urinary tract: Report on six patients. Eur Urol. 2005;48:771-5; discussion 775-778.

[34] Salzberg M, et al. Trastuzumab (Herceptin) in patients with HER-2-overexpressing metastatic or locally advanced transitional cell carcinoma of the bladder: Report on 7 patients. Eur J Cancer Oxf Engl 1990. 2006;42:2660-1.

[35] Hussain MH, A. et al. Trastuzumab, paclitaxel, carboplatin, and gemcitabine in advanced human epidermal growth factor receptor-2/neu-positive urothelial carcinoma: Results of a multicenter phase II National Cancer Institute trial. J Clin Oncol Off J Am Soc Clin Oncol. 2007;25:2218-24.

[36] Oudard S, et al. Multicentre randomised phase II trial of gemcitabine+platinum, with or without trastuzumab, in advanced or metastatic urothelial carcinoma overexpressing Her2. Eur J Cancer Oxf Engl 1990. 2015;51:45-54.

[37] Lapatinib. United States Food and Drug Administration, 2018.

[38] Wülfing C, et al. A single-arm, multicenter, open-label phase 2 study of lapatinib as the second-line treatment of patients with locally advanced or metastatic transitional cell carcinoma. Cancer. 2009;115:2881-90.

[39] Galsky MD, et al. Target-specific, histology-independent, randomized discontinuation study of lapatinib in patients with HER2-amplified solid tumors. Invest New Drugs. 2012;30:695-701.

[40] Cerbone L, et al. Results from a Phase I Study of Lapatinib with Gemcitabine and Cisplatin in Advanced or Metastatic Bladder Cancer: EORTC Trial 30061. Oncology. 2016;90:21-8

[41] Tang S, Dorff TB, Tsao-Wei D, Single arm phase II study of docetaxel and lapatinib in metastatic urothelial cancer: USC trial 4B-10-4.: Journal of Clinical Oncology. 2016; 34(2_suppl):424.

[42] Powles T, et al. Phase III, Double-Blind, Randomized Trial That Compared Maintenance Lapatinib Versus Placebo After First-Line Chemotherapy in Patients With Human Epidermal Growth Factor Receptor 1/2-Positive Metastatic Bladder Cancer. J Clin Oncol Off J Am Soc Clin Oncol. 2017;35:48-55.

[43] Afatinib, United States Food and Drug Administration, 2018.

[44] Choudhury NJ, et al. Afatinib Activity in PlatinumRefractory Metastatic Urothelial Carcinoma in Patients With ERBB Alterations. J Clin Oncol Off J Am Soc Clin Oncol. 2016;34:2165-71.

[45] Tiwari SR, Mishra P, Abraham J, Neratinib A. Novel HER2Targeted Tyrosine Kinase Inhibitor. Clin. Breast Cancer. 2016;16:344-8.

[46] Neratinib, United States Food and Drug Administration, 2018.

[47] Neratinib HER Mutation Basket Study (SUMMIT) - Full Text View - ClinicalTrials.gov. Available at: https://clinical trials.gov/ct2/show/NCT01953926. (Accessed: 27th March 2018)

[48] Hyman DM, et al. Abstract CT001: Neratinib in HER2 or HER3 mutant solid tumors: SUMMIT, a global, multihistology, open-label, phase 2 'basket' study. Cancer Res. 2017;77:CT001-CT001.

[49] NCI-MATCH: Targeted Therapy Directed by Genetic Testing in Treating Patients With Advanced Refractory Solid Tumors, Lymphomas, or Multiple Myeloma - Full Text View - ClinicalTrials.gov. Available at: https://clinicaltr
ials.gov/ct2/show/NCT02465060. (Accessed: 22nd March 2018)

[50] My Pathway: A Study Evaluating Herceptin/Perjeta, Tarceva, Zelboraf/Cotellic, Erivedge, Alecensa, and Tecentriq Treatment Targeted Against Certain Molecular Alterations in Participants With Advanced Solid Tumors Full Text View - ClinicalTrials.gov. Available at: https://cli nicaltrials.gov/ct2/show/NCT02091141. (Accessed: 22nd March 2018)

[51] Bryce AH, et al. Pertuzumab plus trastuzumab for HER2positive metastatic urothelial cancer (mUC): Preliminary data from MyPathway. (2017).

[52] Hayashi T, et al. Targeting HER2 with T-DM1, an Antibody Cytotoxic Drug Conjugate, is Effective in HER2 Over Expressing Bladder Cancer. J Urol. 2015;194:1120-31.

[53] A Study to Determine Best Tumor Response With Trastuzumab Emtansine in Human Epidermal Growth Factor Receptor 2 (HER2) Overexpressing Solid Tumors Tabular View - ClinicalTrials.gov. Available at: https://cli nicaltrials.gov/ct2/show/record/NCT02999672. (Accessed: 23rd March 2018)

[54] Trial of Ado-Trastuzumab Emtansine for Patients With HER2 Amplified or Mutant Cancers - Full Text View - ClinicalTrials.gov. Available at: https://clinicaltrials.gov/ ct2/show/NCT02675829. (Accessed: 23rd March 2018)

[55] Li BT, et al. A multi-histology basket trial of adotrastuzumab emtansine in patients with HER2 amplified cancers. J Clin Oncol. 2018;36:2502.

[56] Study of ADCT-502 in Patients With Advanced Solid Tumors With HER2 Expression - Full Text View ClinicalTrials.gov. Available at: https://clinicaltrials.gov/ ct2/show/NCT03125200. (Accessed: 24th March 2018)

[57] Trastuzumab Deruxtecan (DS-8201a) With Nivolumab in Advanced Breast and Urothelial Cancer - Full Text View - ClinicalTrials.gov. Available at: https://clinicaltrials.gov/ ct2/show/NCT03523572. (Accessed: 7th July 2018)

[58] PRS-343 in HER2-Positive Solid Tumors - Full Text View - ClinicalTrials.gov. Available at: https://clinicaltrials.gov/ ct2/show/NCT03330561. (Accessed: 24th March 2018)

[59] QUILT-3.048: NANT Urothelial Cancer Vaccine: Combination Immunotherapy in Subjects With Urothelial Cancer Who Have Progressed on or After Chemotherapy and PD1/PD-L1 Therapy - Full Text View - ClinicalTrials.gov. Available at: https://clinicaltrials.gov/ct2/show/NCT03197 571. (Accessed: 24th March 2018)

[60] Afatinib Monotherapy in Patients With ERBB-deregulated Metastatic Urothelial Tract Carcinoma After Failure of Platinum Based Chemotherapy - Full Text View - ClinicalTrials.gov. Available at: https://clinicaltrials.gov/ct2/ show/NCT02780687. (Accessed: 24th March 2018)

[61] Study to Assess the Activity of Molecularly Matched Targeted Therapies in Select Tumor Types Based on Genomic Alterations - Full Text View - ClinicalTrials.gov. Available at: https://clinicaltrials.gov/ct2/show/NCT02795156. (Accessed: 24th March 2018)

[62] Agarwal N, et al. Characterization of metastatic urothelial carcinoma via comprehensive genomic profiling of circulating tumor DNA. Cancer. 2018;124:2115-24.

[63] Barata PC, et al. Next-generation sequencing (NGS) of cellfree circulating tumor DNA and tumor tissue in patients with advanced urothelial cancer: A pilot assessment of concordance. Ann Oncol Off J Eur Soc Med Oncol. 2017;28: 2458-63. 